\title{
International Cardiovascular Research Meeting. Copernicus Award Session
}

Medical Research Journal 2019:

Volume 3, Number 2, 120-127

10.5603/MRJ.a2019.0027

Copyright (C) 2019 Via Medica

ISSN 2451-2591

\section{Abstracts}

\section{The effect of an educational campaign on stroke symptoms recognition among primary school kids}

Aleksandra Drozdowska', Jakub Erdmann ${ }^{1}$, Karolina Głowacka ${ }^{1}$, Martyna Moczydłowska', Oskar Puk , Anna Rembisz ${ }^{1}$, Adrian Szkudlarek ${ }^{1}$, Grzegorz Kozera ${ }^{2}$

${ }^{1}$ Students Scientific Association at Department of Neurology, Ludwik Rydygier Collegium Medicum of Nicolaus Copernicus University, Bydgoszcz, Poland

2Department of Neurology, Ludwik Rydygier Collegium Medicum of Nicolaus Copernicus University, Bydgoszcz, Poland

Background: Stroke is the third leading cause of disability among adult patients. Poor awareness regarding stroke emergencies and symptoms limits its effective therapy. School promotion of the essential knowledge about stroke manifestations may be beneficial, however little is known about its efficacy among Polish pupils. Therefore we aimed asses the effects of education campaign on of stroke knowledge among primary school kids.

Methods: Dedicated educational campaign entitled "uDARuj Zdrowie" including 45 minutes lesson with movie presentation has been performed among pupils from III class of primary school. Kids answered the questionnaire of: 9 one-choice questions concerning stroke awareness and first aid procedures and 1 question regarding typical stroke symptoms before and $+/-12$ months after the lesson.

Results: 287 kids participated in lessons, and 145 responded the questionnaire after the follow up. Median of correct answers regarding stroke awareness and first aid procedures were $5(0-5)$ before and $6(1-9)$ after the follow up $(p<0.01)$, including: cause of a stroke $32.12 \%$ vs. $37.41 \%$ ( $p<0.01$ ), immediate call for ambulance $62.21 \%$ vs. $82.44 \%(p<0.01)$ and therapeutic time window $89.21 \%$ vs. $95.83 \%$ ( $p<0.01$ ). Median of recognized stroke symptoms was 2 before and 4 after the follow up $(p<0.01)$. Conclusions: Dedicated educational campaign improves kids knowledge about stroke symptoms and procedures. Basic stroke education should be implemented in primary education.

\section{Predictors of an early diagnosis of total occlusion of infarct-related artery in patients with non-ST-elevation myocardial infarction (NSTEMI)}

Irmina Antonina Morawska, Rafał Niemiec, Katarzyna Mizia-Stec

Leszek Giec Upper-Silesian Medical Centre, 1st Chair and Clinic of Cardiology, Medical University of Silesia in Katowice

Background: Some patients with non-ST-elevation myocardial infarction have a total occlusion (TO) of infarct-related artery (IRA) Thereupon, acute coronary occlusion (ACO) may also present as non-ST-elevation myocardial infarction (NSTEMI) and thus veil the real threat. The prevalence and impact of TO of IRA on outcomes in patients with NSTEMI remain unclear. The purpose of the study was to analyse the incidence of total occlusion of IRA and to assess the predictors of total occlusion of IRA in patients with NSTEMI. Methods: The study was conducted as a retrospective cross-sectional analysis of 399 consecutive patients with NSTEMI (M = 293, mean age $70.3 \pm 10.1$ ) hospitalised and treated by primary percutaneous coronary intervention $(\mathrm{PCl})$ in the 1st Chair and Clinic of Cardiology, Medical University of Silesia in Katowice in 2017. The study population was categorized into patients with TO of IRA and with hemodynamically significant culprit coronary lesion (non-TO) on coronary angiography. Demographic and clinical data, including laboratory test results and 
electrocardiographic parameters, were acquired by means of meticulous review of discharge summaries and electronic health records. TO of IRA, in-hospital and 12-month mortality were the outcomes.

Results: TO was found in 138 (34.6\%) patients with NSTEMI. Patients with TO had a greater incidence of diabetes mellitus $(p=0.003)$ and atrial fibrillation $(p=0.02)$. Coexistence of serum triglicerides level $(p=0.001)$, glucose level $(p=0.0005)$ and baseline troponin level $(p=0.004)$ corresponded with the highest incidence of TO of IRA. The left circumflex artery (LCX) was the major IRA in the TO group (39.1\%), whereas the left anterior descending artery (LAD) was more commonly the IRA in the non-TO group (48.5\%). Multivariate analysis revealed that LCX as the culprit lesion (OR 0.53 [0.41-0.68], $\mathrm{p}<0.0001$ ) was an independent predictor of IRA flow in TIMI scale. In-hospital and 12-month mortality were significantly higher in the TO group than non-TO group ( $2.8 \%$ vs. $1.1 \%, p=0.007$ and $6.1 \%$ vs. $2.5 \%, p=0.0003$ ). Conclusions: The identification of NSTEMI patients with total occlusion of infarct-related artery is challenging. In the population of patients with NSTEMI, TO of IRA represents a considerably frequent phenomenon and corresponds with established clinical markers of impaired outcome. These patients may require different clinical approach than typical NSTEMI patients. Therefore, the utmost caution should be paid to prevent delay of coronary angiography in NSTEMI patients with higher baseline troponin levels and metabolic disturbances who represent the increased risk of acute coronary occlusion.

\title{
3. The use of body composition analysis in diagnosing heart failure - comparison with brain natriuretic peptide
}

jakub Rzeszuto ${ }^{1}$, Izabela Garbacz ${ }^{1}$, Maciej Lemański1, Wioleta Stolarek², Michał Siedlaczek ${ }^{2,3}$, Łukasz Pietrzykowski ${ }^{3}$, Piotr Michalski ${ }^{3}$

${ }^{1}$ Students' Scientific Society, Department of Cardiology and Internal Medicine Nicolaus Copernicus University in Torun. Collegium Medicum in Bydgoszcz, Poland

2Department of Cardiology and Internal Medicine Nicolaus Copernicus University in Torun. Collegium Medicum in Bydgoszcz, Poland ${ }^{3}$ Department of Health Promotion Nicolaus Copernicus University in Torun. Collegium Medicum in Bydgoszcz, Poland

Background: Heart failure (HF) concerns 26 million people around the globe and is one of major causes of hospitalisations in Europe. Currently, one of the recommended by European Society of Cardiology (ESC) methods of screening is checking the brain natriuretic peptide (BNP) level. It is a very non-specific test however, as its increase may also occur due to left ventricle hypertrophy, hyperthyroidism, renal failure or other causes. This made us look for a way that is faster, cheaper and would allow to detect HF earlier, thus giving opportunity to start the treatment in an earlier stage, when prognosis is better. To do so we have tried to prove relation between body water content and presence of HF. Methods: The approach we are exploring is use of body content analysis done thanks to bioimpedance, since other studies show close relation between percentage of water in a body mass and management of acute HF and this is a good non-invasive method to determine that factor. Study group consists of hospitalised stable adult patients admitted for diagnostics towards HF detection. During the hospitalisation the necessary diagnostic tests were performed and additionally body content analysis using bioimpedance method. During the hospitalisation laboratory tests and imaging examinations were performed as required for diagnostic and treatment purposes, as well as body content analysis using bioimpedance method specifically for the study. Twenty seven patients were encircled to the study, with total of 100 planned. Results: According to the current data from the group of 27 patients, there was a significant negative correlation between BNP and BMI $(R=-0.529$, [i] $p=0.005$ [/i]) and a significant positive correlation between BNP and fat-free tissue $(R=0.549$, [i] $p=0.004[/ i])$, total water $(R=0.463$, [i] $p=0.017$ [/i]) and extracellular $(R=0.576$, [i] $p=0.002$ [/i]). Increased BNP level was associated with left ventricular dysfunction ([i] $p=0.022[/ i]$ ) and atrial fibrillation ([i] $p=0.025[/ i]$ ). There was no correlation between body content and left ventricular ejection fraction (LVEF). Furthermore, there was no relation between body content and systolic or diastolic left ventricular dysfunction. Conclusions: Current results indicate bioimpedace body content analysis may be a method to assist in the diagnosis of HF. However, it is necessary to analyze results from the planned group of patients. At this point basic limitations of our research are monovariable analysis and small count of the study group.

\section{The effect of Homocysteine on fibrinolytic and proteolytic parameters depends on the intraluminal thrombus thickness}

\author{
Monika Adamowicz, Natalia Grześ, Aldona Siennicka
}

Department of Laboratory Diagnostics, Pomeranian Medical University of Szczecin.

Background: Increased levels of homocysteine (Hcy) have been implicated as risk factor for cardiovascular disease and there is some evidence that may be associated with an increased risk of having an abdominal aortic aneurysms (AAA). Homocysteine was suggested to enhance the proteolysis as well as disturbance of the coagulation/fibrinolysis system, which in turn may promote local proteolytic degradation of the aortic wall. In the majority of cases, the aneurysmal walls are covered by the intraluminal thrombus (ILT). It was suggested that depending on the thickness, ILT shows the significant differences in the activity of proteolytic and fibrinolytic processes, which emphasizes that such peculiar structure may influence the pathogenesis and development of the disease. The aim of this study was therefore to investigate the effect of incubation of homocysteine on proteolytic and haemostatic activity in individual aneurysm compartments. 
Methods: Aneurysmal aortic specimens were obtained from 36 patients (27 men and 9 women), who were diagnosed with the presence of both thick $(\geq 25 \mathrm{~mm})$ and thin $(\leq 10 \mathrm{~mm})$ intraluminal thrombus in one aneurysm sac. The obtained aneurysm tissue samples were divided into four groups: thick thrombus; thin thrombus; wall adjacent to thick thrombus; wall adjacent to thin thrombus. In a series of experiments, the thick and thin ILT as well as adjacent walls were separately incubated in medium, in the presence of DL-homocysteine $(100 \mu \mathrm{mol} / \mathrm{L}$ or $500 \mu \mathrm{mol} / \mathrm{L})$. Untreated aneurysm fragments were used as controls. After treatment, tissue samples were collected for protein extraction and the concentration of t-PA (ng/mg,) and TF $(\mathrm{pM} / \mathrm{mg})$ were determined using immunoenzymatic test (ELISA). The MMP-2 $(\mu \mathrm{M} / \mathrm{mg})$ activity was performed using fuorescence resonance energy transfer (FRET) assay. Results: Incubation of thin thrombus fragments with DL-Hcy resulted in an increase of t-PA concentration compared to control tissue (1.39 \pm 1.65 vs. $0.84 \pm 0.74, p=0.024)$. Whereas, in thick ILT sections, MMP-2 activity as well as t-PA concentrations was decreased (respectively, $2.53 \pm 2.02$ vs. $3.28 \pm 2.65, p=0.006 ; 0.67 \pm 0.57$ vs. $0.96 \pm 0.91, p=0.021$ ). Moreover, DL-Hcy induced the activity of MMP-2 in the wall underlying thin IL T in comparison to control tissue (9.54 \pm 5.88 vs. $7.44 \pm 4.48, p=0.011)$. In turn, in wall adjacent to thick thrombus the activity of both MMP-2 and TF were decreased in contrast to controls (respectively, $5.89 \pm 3.39$ vs. $7.26 \pm 5.49, p=0.046$; $67.13 \pm 72.59$ vs. $114.46 \pm 106.29, p=0.007)$.

Conclusions: These data demonstrate that homocysteine increases the activity of both haemostatic and proteolytic parameters in thinner AAA segments, which suggested that Hcy may enhance damage of arterial wall in this part of aneurysm. Those results might be significant for future AAA studies in the context of predictive tools of disease severity, risk of rupture as well as potential targeted therapy.

\section{The importance of focal adhesion in coronary artery endothelial cell activation and barrier function}

\section{Maciej Gagat ${ }^{1}$, Wioletta Zielińska ${ }^{1}$, Marta Hałas-Wiśniewska ${ }^{1}$, Dariusz Grzanka², Alina Grzanka1}

${ }^{1}$ Department of Histology and Embryology, Faculty of Medicine, Nicolaus Copernicus University in Torun, Collegium Medicum in Bydgoszcz, Bydgoszcz, Poland

2Department of Clinical Pathomorphology, Faculty of Medicine, Nicolaus Copernicus University in Torun, Collegium Medicum in Bydgoszcz, Bydgoszcz, Poland

Background: Maintaining the integrity of the endothelial barrier is a prerequisite for homeostasis. This is possible due to the involvement of proteins that sustain normal cell-cell and the cell-extracellular matrix (ECM) contacts. In the case of the endothelium, the basic protein involved in adherence junctions is VE-cadherin, while talin is closely associated with the focal adhesion. It is postulated that in the endothelial cells mainly talin-1 is expressed, whereas talin-2 homolog is characteristic for cardiac muscle cells. It is possible, however, that talin-2 may also occur in endothelial cells, but its role remains unknown. The aim of the study was to elucidate the involvement of both talin homologues and focal adhesion in the inflammatory response of vascular endothelial cells. Methods: We used primary human coronary artery endothelial cells (pHCAECs) derived from healthy 23-years old white male treated with tumour necrosis factor alpha (TNF $\alpha$ ). Endothelial cells were activated with $100 \mathrm{nM}$ concentration of TNF $\alpha$. Tropomyosin-1 (TPM1) expression was regulated using the CRISPR/Cas system. Alterations in cell-cell and cell-ECM contact were confirmed by fluorescence labeling of junctional and focal adhesion proteins together with the actin cytoskeleton. Proteins levels were assessed using western blot technique. The angiogenic potential was investigated using in vitro tube formation assay. All results obtained on the fixed cells were analyzed in relation to those from livecell imaging. Morphology of migrating cells was investigated using correlated fluorescence and holotomography microscopy.

Results: TNF $\alpha$-activated pHCAECs change F-actin organization pattern from star-like into linear stress fibres. Simultaneously, $\mathrm{TNF} \alpha$ induces formation of angiogenic-dependent migrasome-like structures, which can be released to the medium and stay in the place of previous strong cell-cell junction, attached to the place of strong focal adhesion site or taken up by surrounding cells. This suggests their role in cell communication. These changes can be prevented by stabilizing the actin cytoskeleton structure. Stabilization of actin filaments was achieved using CRISPR systems regulating the naïve expression of TPM1. We showed that activation of TPM1 expression dismissed inflammatory response of pHCAECs maintaining endothelial integrity and inhibiting in vitro tube formation, whereas knockout of TPM1 increased the inflammatory response of pHCAECs leading to loss of endothelial integrity and enhanced in vitro tube formation. Furthermore, our experiments showed that activation of TPM1 inhibits focal adhesion of $\mathrm{pH}$ CAECs, even after their activation with TNF $\alpha$. On the other hand, knockout of TPM1 enhanced focal adhesion of TNF $\alpha$-activated pHCAECs. It resulted in the increased expression of talin-2 and vinculin but not talin-1. Moreover, the activation of TPM1 inhibited cleavage of VE-cadherin in pHCAECs, even after their activation with TNF $\alpha$ and promoted $\alpha$-catenin-based stabilization of cell-cell junction continuity, especially in TNF $\alpha$-activated cells, while knockout of TPM1 induced high expression of $\beta$-catenin in TNF $\alpha$-activated pHCAECs. Conclusions: TNF $\alpha$-induced activation of pHCAECs leads to F-actin cytoskeletal rearrangement promoting angiogenesis. CRISPR systems regulating the expression of TPM1 can be used to control F-actin stabilization and endothelial integrity by the maintenance of both focal adhesion and cell junctions. The increase in talin-2 expression in cells with actin cytoskeleton rearrangement may indicate the involvement of this protein in the migration of activated endothelial cells due to enhanced traction force. However, the activation of TPM1 expression dismisses the inflammatory response of pHCAECs and protects endothelial integrity, while the knockout of TPM1 leads to cellular changes characteristic for angiogenesis. 


\title{
6. Science-fiction or the future of medicine: how using all-day cardiological telemonitoring can help diagnose the coronary heart disease
}

\author{
Katarzyna Fąderska, Katarzyna Jarzębska, Aleksandra Bąk, Aneta Kida, Tomasz Cyganek, Maciej Drwęcki
}

Biostatistics Scientific Club at the Cathedral and Epidemiology Facility, Medical University of Silesia

Background: Advancement in healthcare technologies, such as tele-ECG provides easier and faster medical contact for cardiology patients. Tele-ECG followed by telephone consultation should identifies first signs of heart diseases exacerbation, resulting in better clinical outcomes. However, there is lack of data comparing efficiency of telemonitoring in patients after percutaneous coronary procedures. Therefore, the aim of this study was to evaluate long term clinical outcomes between patients with or without telemonitoring support. Methods: This is a multicentre, retrospective registry of 400 consecutive patients, who undergone percutaneous coronary procedure (PCl) from 10.2016 to 06.2018. 202 of them were provided with teleECGdevice and telephone consultations, while 198 patients did not agree for telemonitoring. Patients with both acute coronary syndrome and stable angina were included. The average time of telemonitoring was 312 days. Results: At baseline, patients in monitored group were more often presented with myocardial infarction ( $50 \%$ vs. $35 \% ; p=0.01$ ), especially STEMI (18\% vs. $9 \% ; p<0.01)$. After one year, the incidence of hospitalization due to myocardial infarction (3.8vs. $4.7 \% ; p=0.16)$ and all couse death were numerically lower in monitored group, however the difference was not statistically significant. The rate of hospitalizations with heart failure exacerbation were significantly lower in monitored group ( $2 \%$ vs. $4.10 \% ; p=0.04$ ). The incidence of arrhythmias and rePCI/CABG were significantly more common in study group ( $7.4 \%$ vs. $2 \% ; p<0.05$ and $9 \%$ vs. $5 \% ; p=0.04$ respectively) were more often in monitored group. Conclusions: Our study suggests that exacerbation of heart disease in patients supported with telemonitoring were diagnosed earlier. It might result in lower incidence of more serious medical conditions. Further studies with larger and more comparable groups are needed to proof this hypothesis.

\section{Knowledge and adherence of patients on warfarin therapy toward their treatment regimen in Alshaab and Ahmed Gasim Hospitals, Sudan, 2018}

\section{Lina Nasr}

5th year medical student, Khartoum University

Background: Warfarin is commonly used oral anticoagulation which is needs to be continuously monitored both clinically and laboratory due to its narrow therapeutic index and potentially life threatening complications. this study aims to assess knowledge and adherence of patients on warfarin therapy toward their treatment regimen and to identify barriers that prevent patients to take their warfarin therapy regulary. Methods: A cross-sectional survey was conducted at Alshaab teaching hospital heart section and Ahmed Gasim hospital Khartoum, Sudan. The systematic random sampling method was used in sample selection and interview based questionnaires were used to determine the socio-demographic characteristics, the patient's knowledge of warfarin therapy and medication adherence.

Results: Male patients (52.5\%) exceeded females (44.8\%), the mean age was 48.79 . About $68.4 \%$ of the studied patients received education regarding Wafarin Oral anticoagulant by their physician. $43 \%$ of the studied patients had moderate overall knowledge score, while more than half of them had either satisfactory or good adherence levels (62.2\%). There were negative significant correlations between patients' adherence to Warfarin Oral Anticoagulant and their level of knowledge $\left(r=0.035^{\star}\right)$. There were significant differences between patient's education, sex, occupation and their level of knowledge, whereas no significance differences between patient's sex/age and their level of adherence were found. There were three barriers prevent the patients from taking their medication. Forgetting (43.7\%) was found to be the main reason followed by drug unavailability (36.8\%) and high cost (19.5\%) respectively.

Conclusions: The majority of the studied patients had moderate overall knowledge score about Warfarin Oral Anticoagulant, and more than half of them had either satisfactory or good adherence levels. There were negative significant correlations between patients' adherence to Warfarin Oral Anticoagulant and their level of knowledge.

\section{Selected haemostatic factors in patients with abdominal aortic aneurysm}

Natalia Grześ, Monika Adamowicz, Siennicka A.

Department of Laboratory Diagnostics, Pomeranian Medical University of Szczecin.

Background: Abdominal aortic aneurysm (AAA) has increasingly been recognized as a significant cause of mortality in older adults. It is suggested that the mechanism contributing to aneurysm rupture is the imbalance between the fibrinolytic system and the activation of the coagulation system. In many research, haemostatic markers were examined for the association with the AAA growth, size of AAA, as well as ILT size. However, the results are inconsistent. The aim of our study was the evaluation of selected haemostatic markers in patients with AAA and controls; and evaluation of the correlation with AAA diameter and thrombus thickness.

Methods: The study included 36 patients with AAA, treated in the Department of Vascular and General Surgery and Angiology of PUM in Szczecin, and 30 controls with normal diameter $(<3 \mathrm{~cm})$. Venous blood samples were obtain from control group and each patient with AAA 
before elective repair of aneurysm. Whole blood was collected into tubes with plasma citrate to assay D-dimer and tissue plasminogen activator (t-PA). Whereas plasminogen activator inhibitor-1 (PAl-1) was measure in CTAD plasma. The ELISA or ELFA method were used to determine the concentration of all factors.

Results: Concentrations of D-dimer (ng/mL) and t-PA (ng/mL) were significantly higher in patients with AAA (respectively: $2673 \pm 1824 \mathrm{vs} .1125 \pm 895$, $p=0.002 ; 5.2 \pm 2.5$ vs. $4.2 \pm 1, p=0.039)$. PAl -1 ( $\mathrm{ng} / \mathrm{mL})$ levels did not show a significant difference $(12.0 \mathrm{vs} .13 .0 ; \mathrm{p}=0.586)$. The correlation showed negative relationship between t-PA and ILT thickness $(r=-0.53 ; p=0.001)$ and t-PA and aneurysm diameter $(r=-0.38 ; p=0.023)$. Conclusions: The higher plasma concentrations of D-dimer and t-PA may support the hypothesis that the secondary fibrinolysis plays an important role in the pathogenesis of the AAA formation and strong negative correlation between t-PA concentration and ILT thickness, suggests that coagulation-fibrinolysis imbalance may accelerate ILT formation and possibly aneurysm progression.

\title{
9. A pilot study of chromogranin B in patients undergoing off-pump coronary artery bypass grafting
}

\author{
Karolina Malec ${ }^{1 *}$, Iwona Mazur ${ }^{*}$, Artur Słomka ${ }^{2}$, Joanna Boińska ${ }^{2}$, Mariusz Kowalewski ${ }^{3}$, Wojciech Pawliszak ${ }^{4}$, \\ Joanna Banach ${ }^{5}$, Ewa Żekanowska ${ }^{1}$
}

*Both authors contributed equally to this work

${ }^{1}$ Student Scientific Association of Laboratory Hematology, Department of Pathophysiology, Nicolaus Copernicus University in Toruń, Ludwik Rydygier Collegium Medicum in Bydgoszcz, Poland

${ }^{2}$ Department of Pathophysiology, Nicolaus Copernicus University in Toruń, Ludwik Rydygier Collegium Medicum in Bydgoszcz, Poland

${ }^{3}$ Clinical Department of Cardiac Surgery, Central Clinical Hospital of the Ministry of Interior in Warsaw, Poland

4 Department of Cardiac Surgery, Dr Antoni Jurasz Memorial University Hospital in Bydgoszcz, Poland

${ }^{5}$ Second Department of Cardiology, Nicolaus Copernicus University in Toruń, Ludwik Rydygier Collegium Medicum in Bydgoszcz, Poland

Background: Chromogranin B (CgB) belongs to a family of acidic proteins called granins, that are widely distributed in many cell types. It has been shown that $\mathrm{CgB}$ is also produced by the ventricular myocardium and can become a potential biomarker of heart failure. The clinical value of $\mathrm{CgB}$ for evaluating myocardal disease progression or treatment response is still poorly understood. The aim of the study was to assess CgB concentrations in patients undergoing off-pump coronary artery bypass grafting (OPCABG).

Methods: The study included 30 male patients (mean age 63) with coronary artery disease (CAD) who underwent elective, first-time isolated OPCABG and 20 healthy, nonsmoking, age-matched males (mean age 59). Patients with left ventricular ejection fraction (LVEF) < 40\% were excluded. Venous blood samples from patients were collected twice: on the day of surgery and seven days later. CgB concentrations were evaluated by enzyme-linked immunosorbent assay.

Results: CgB concentration in patients scheduled for surgery was similar to observed in healthy males $(\mathrm{Me}=77.04 \mathrm{ng} / \mathrm{mL} \mathrm{vs} . \mathrm{Me}=82.56 \mathrm{ng} / \mathrm{mL}$, $\mathrm{p}=\mathrm{NS})$. CgB concentrations 7 days after OPCABG were significatly higher than in the day of surgery $(\mathrm{Me}=87.54 \mathrm{ng} / \mathrm{mL} \mathrm{vs} . \mathrm{Me}=77.04 \mathrm{ng} / \mathrm{mL}$, $\mathrm{p}=0.004$ ). We found no difference in CgB concentrations, depending on the surgical procedure: no-touch OPCABG vs. touch (traditional) OPCABG. Nevertheless, CgB levels in patients after touch surgery were higher (Me $=88.73 \mathrm{ng} / \mathrm{mL}$ ) than in patients after no-touch surgery $(\mathrm{Me}=82.01 \mathrm{ng} / \mathrm{mL}$ ). Additionally, there were no differences in $\mathrm{CgB}$ concentrations in subgroups of patients divided according to the presence of diabetes mellitus, hypertension, dyslipidemia or cigarette smoking. We found no relations between CgB concentrations and age, body mass index (BMI), procedure duration and number of grafts.

Conclusions: Off-pump coronary artery bypass surgery increases circulating CgB concentrations, regardless of the surgical technique. This phenomenon may be associated with the release of $\mathrm{CgB}$ from ventricular myocardium and deserves further investigations.

\section{ECG-based apnea-hypopnea index in acute ischemic stroke patients}

\section{Joanna Bisaga, Daria Siewior, Magdalena Kurczab, Wojciech Kowalczyk, Klaudia Redel, Klaudia Adamczewska}

Medical University of Silesia in Katowice,1st Chair and Clinic of Cardiology in Upper Silesian Medical Centre

Background: Obstructive sleep apnea (OSA) is a breathing disorder during sleep regarded as cardiovascular risk factor associated with increased mortality. The Apnea-Hypopnea Index (AHI) is represented by the number of apnea and hypopnea events per hour of sleep. ECG seems to be potentially the most practical, non-invasive tool for screening for sleep disordered breathing (SDB). In patients with AHI > 15 risk of SBD is significant. OSA is important, modifiable risk factor of acute ischemic stroke (AIS) which is responsible for majority of neurological disabilities in adults. The aim of our study was to estimate AHI based on ECG in patients with cryptogenic AIS and in healthy subjects. We wanted to measure if the episode of AIS leads to increased AHI.

Methods: The study group consisted of 92 patients diagnosed with first symptomatic cryptogenic AIS (age: $60 \pm 14 ; 51 \%$ males) hospitalized in Neurology Department, Leszek Giec Upper-Silesian Medical Centre of the Silesian Medical University in Katowice in years 2015-2018. Patients were divided into 2 groups: TACI (anterior ischemia) and non-TACI (posterior ischemia). Each patient had 7-days Holter recording. Control group contained 50 healthy people (age: $55 \pm$ 9; 66\% males) after Holter ECG monitoring in 1st Chair and Clinic of Cardiology in Upper Silesian Medical Centre in Katowice. Data was analyzed and automated algorithm was used to estimate patients' AHI. AHI thresholds of $<5$ and $>15$ were used to indicate low and high probability of OSA. 
Results: AHI was significantly increased in AIS patients in comparison to control group ( $p=0.001)$. AHI in non-TACI patients (14 \pm 5.3$)$ was higher than in TACI $(10.5 \pm 4.8)$ and control group $(8.5 \pm 8.4)$. AHI $>15$ occurred in $29 \%$ AIS patients and in $16 \%$ from control group Conclusions: Patients after AIS are more likely to have higher AHI and higher probability of OSA. Non-TACI AIS is significantly more predisposing factor of SDB which might be related to localization of ANS respiratory center.

\title{
11. How do the patients with thoracic aortic dissection assess their quality of life after bentall procedure?
}

\author{
Joanna Bisaga, Monika Kasperczak, Bartosz Kończyk
}

Department of Cardiac Surgery, Leszek Giec Upper-Silesian Medical Centre of Silesian Medical University in Katowice-Ochojec, Katowice, Poland

Background: Post-surgical quality of life is an important factor in every post-operative patient management. It affects not only patients but also clinical practice in making treatment decisions. The aim of the study was to assess the short-term and longterm quality of life of the patients with acute thoracic aortic dissection who underwent Bentall procedure between January 2012 and January 2018. We wanted to measure the effects of disease and medical intervention on the individuals' attitude towards future life. Methods: We gathered data from medical records of 218 patients from Department of Cardiac Surgery, Leszek Giec Upper-Silesian Medical Centre of Silesian Medical University in Katowice-Ochojec, diagnosed with ICD-10: 171.0 [Dissection of aorta (any part)] between January 2012 and January 2018. After excluding patients with Marfan disease and reoperation, we contacted patients, who survived the surgery and were still alive. We managed to contact and interview 40 patients, who were in different time after surgery. In March 2018 they filled in The World Health Organization Quality of Life Questionnaire (BREF). WHOQOL-BREF consists of 26 questions, due to measure the following domains: physical health, psychological health, social relationships and environment. We compared the similarities and differences corresponding to the number of years after the operation.

Results: The results show that the average patient after the Bentall procedure assessed the physical health lower and social relationships higher than the rest of the measured aspects. Women scored their quality of life higher than men in all age groups. Pain prevents patients from everyday activities to little extent. They are rather satisfied with their sleep. Moreover, they are content with access to health services. They seldom feel anxious or depressed.

Conclusions: Patients are very content with their social relationships and support from their family and friends. They are much less satisfied with their work capacity and physical condition, which is corelated with lower subjective qality of life after the surgery. After the operation they became more dependent on health care services and they need regular medical check-ups. However, they believe that their life is meaningful and they value their life more than before the surgery.

\section{Extracellular matrix cylinder reconstruction of tricuspid valve in patient with dual-chamber implantable cardioverter-defibrillator (ICD-DDD)}

\section{Kinga Panasiewicz ${ }^{1}$, Przemysław Szałański²}

${ }^{1}$ Medical University of Lodz, Faculty of Medicine

${ }^{2}$ Military Institute of Medicine

Background: Extracellular matrix valve protheses have been getting more attention in recent years, for their advantage over the other commonly used materials. Bioscaffolds, by inducing the host tissue growth, reduce significantly thrombogenicity and inflammatory foregin-body response of the organism, show a significant antibacterial activity, as well as they are more resitant to calcification and overall degradation. Therefore extracellular matrix is a reasonable option for high-risk patients with various co-exhisting conditions. This case concerns a patient diagnosed with a severe tricuspid regurgitation found after the dual-chamber implantable cardioverter-defibrillator (DDD-ICD) replacement procedure.

Methods: 65-year old male patient was urgently admitted to the cardiac surgery unit with a severe tricuspid regurgitation after the dual chamber implantable cardioverter-defibrillator (DDD-ICD) replacement procedure. The patient was immidiately qualified for TVR surgery. Tricuspid valve native cusps were resected and tubular prothesis made of small intestinal submucosa-derived extracellular matrix (ECM) was subsequently implanted. 12-month follow-up observations of patient's conditions have been collected, including echocardiographic examinations.

Results: Post-operative echocardiogram showed a proper function of the cylinder ECM tricuspid valve, with a small thin central jet, without any leakage around the prothesis. The patient was discharged from the hospital 9 days later in good condition, without neurological deficits, cardiovascularly and respiratorily stable. 12 months after the surgery echocardiographic examination shows the same findings; the patient leads a normal life and has no problems with executing everyday tasks.

Conclusions: Extracellular matrix cylinder reconstruction can be successfully performed in patients with ICD-DDD, without damaging the electrode passing through the heart chambers. The material that was used for the reconstruction is a very good option for high-risk patients with many comorbidities or foreign bodies inside the heart (stents, electrodes), in order to reduce thrombogenicity, calcification, acute immune response or bacterial infections. 


\section{Broken heart syndrome - a case study of 67-years-old female cancer patient}

Joanna Bisaga, Marcelina Cieślik, Aleksandra Bąk, Tomasz Cyganek, Katarzyna Jarzębska

Medical University of Silesia in Katowice, Biostatistics Scientific Club at the Cathedral and Epidemiology Facility, Center for Cardiovascular Research and Development, American Heart of Poland

Takotsubo cardiomyopathy, known also as Broken Heart Syndrome, is a rare acute heart failure syndrome affecting 2-3\% of people. 90\% occurs in women, mostly postmenopausal. It can be induced by physical or emotional trigger factor.

67-years-old woman with the history colon cancer (2016) was admitted to surgical ward with sub-obstructive symptoms (26.05.2017). Subsequently laparotomy was performed. During surgery patient needed continuous iv catecholamines infusion due to hypotonia. After the surgery, she demonstrated acute circulatory and respiratory failure, cardiac arrest (VF mechanism) and cardiogenic shock. She was transferred to cardiology ward with diagnosis of ST-elevation myocardial infarction (STEMI) of anterior wall. Blood tests showed raised cardiac biomarkers: CK-MB, Troponin T. Coronary angiography did not reveal significant stenosis in coronary arteries. Pulmonary embolism was excluded in angio-CT. In transthoracic echocardiography (TTE) there was generalized hypokinesis with LV EF reduced to 10-15\%. Due to cardiogenic shock, Intra-aortic balloon pump (IABP) was utilized. Patient was diagnosed with MI type 2 and transferred to Intensive Care Unit. Four weeks later, in control TTE, the LV EF was $60 \%$ with mild mitral and trigeminal valve regurgitation. During following months, the recurrence of cancer was diagnosed and she received oncological treatment. At one-year follow up, woman reported fatigue, dizziness, malaise and presyncope symptoms. Holter-ECG performed in outpatient clinic, revealed short episodes of 2nd degree AV block, Mobitz II and she was qualified for pacemaker implantation. The patient had urine stagnation (09.2018). Histopathological exam showed urothelial cancer. Recently, her cardiology status remains stable and she continues therapy.

Takotsubo cardiomyopathy is characterized by symptoms imitating acute coronary syndrome, so it can be easily mistaken and treated as such in the initial period. In this case the trigger factor could be surgical procedure, elevated catecholamines, serious illness(cancer), chemotherapy or mental stress associated with cancer. The prevalence of Takotsubo cardiomyopathy can be underestimated due to lack of awareness of this rare diagnosis.

\section{Safe inhibition of P-glycoprotein as a potential prolonger of treatment response to levodopa in Parkinson's disease}

\section{Brynjar J Andreassen}

Students' Scientific Society, Department of Pharmacology and Therapeutics, Faculty of Medicine, Collegium Medicum in Bydgoszcz, Nicolaus Copernicus University, Bydgoszcz, Poland.

Parkinson's disease (PD) is a neurodegenerative condition characterized by the progressive loss of dopaminergic motor neurons in the midbrain. Therefore replenishing the depleted dopamine supply in the brain is the target of current PD-directed therapies. As exogenous dopamine cannot cross the blood-brain barrier, levodopa, a precursor of dopamine that can permeate this barrier, is the drug of choice for treating PD patients. However, only $5-10 \%$ levodopa is available for the neurons as the rest is effluxed by P-glycoprotein, an ATP-dependent transporter of the multidrug resistance family of transporters. Indeed, P-glycoprotein-mediated efflux is the cause of multidrug resistance in several diseases, including cancer. Therefore, current therapies are being designed to overcome the drug efflux, for example, by using other competitive substrates of the transporter such as verapamil.

In this review, we have discussed PD, the blood-brain barrier, and the ongoing research on improving the bioavailability of levodopa in the brain, while considering the potential drawbacks of these approaches.

We believe that this will provide a comprehensive picture of the current scenario regarding PD treatment and highlight the scope for improvement.

\section{The current approach of arterial hypertension based on recent recommendation of ESC}

\section{Elda Ahmeti}

Student Medical University of Tirana

Background: Arterial hypertension is one of the most important and modifiable risk factors for cardiovascular disease in Europe and worldwide. As a major contributor to worldwide morbidity and mortality, it poses a massive socioeconomic burden. Blood pressure control is still inadequate in most of the hypertensive patients $(<140 / 90 \mathrm{~mm} \mathrm{Hg}$ ) even though great progress in perception, diagnosis and treatment of hypertension is made. The intention of this research paper consists on analyzing and comparing guidelines through the current years in order to generate an updated definition, diagnostics and treatment about arterial hypertension regarding the population standards. Performing a literature search for new evidence on hypertension in general would inform for the reaffirmation of the current guidelines used in our hospitals.

Methods: The PubMed and ESC/ESH guidelines were searched. The searches were limited to articles of adult ages published between 2013-2018 in English language. The studies were reviewed and controlled to get the best extract. Determination of risk factors, lifestyle and demographic characteristics tends to give a full overview of the patient and define the proper treatment scheme. 
Results: In a comparison, the current guidelines refer more lifestyle modifications especially in uncomplicated hypertension. Drug treatment is considered afterwards depending on hypertension grade with its main purpose to lower blood pressure. By how much the blood pressure should be lowered is currently a matter of controversy. The 2013 European and German national guidelines recommend a target blood pressure of $<140 / 90 \mathrm{~mm} \mathrm{Hg}$ for most patients. The 2018 European Society of Hypertension guideline recommends as the first objective lowering blood pressure $<140 / 90 \mathrm{~mm} \mathrm{Hg}$ for all patients and afterwards hypertension target values should be $<130 / 80 \mathrm{~mm} \mathrm{Hg}$ for most patients.

Conclusions: The most efficient treatment of hypertension is reached knowing your patient, classifying the level of hypertension and determine risk factors for cardiovascular disease. As it always starts with lifestyle modifications, the drug treatment preferably starts with a two drugs combination except the hypertension Grade I. As an addition to resistant hypertension treatment is a low dose of spironolactone or another diuretic therapy like higher dose of thiazides or a loop diuretic or bisoprolol.

\section{Improvement of the quality of resuscitation among students and doctors after applying electrodes with a pressure sensor}

\section{Szankin Z, Bruska M}

Students' Scientific Society, Department of Cardiology and Internal Medicine, Nicolaus Copernicus University in Torun. Collegium Medicum in Bydgoszcz, Poland

Background: Correct chest compressions significantly increase the coronary perfusion pressure, which increases the chance of survival of patients. High quality CPR is carried out in accordance with the recommendations, i.e. with a depth of compressions of 5-6 cm and a frequency of 100-120 per minute. During the study, we focused on two elements: depth and the frequency of compressions.

Methods: 96 people participated in the study: students of CM UMK in Bydgoszcz (37 people) and doctors from No. 1 Jurasz University Hospital in Bydgoszcz (59 people). Defibrillators equipped with real-time monitoring of the quality of pressure were used. Every participant in this study initially began to compress the manikin with which the electrode with the pressure sensor was used, but without feedback. Then he proceeded to oppress the second manikin, which also possessed an electrode with a pressure sensor, from which he received real-time feedback on the depth of pressure of the chest, with what frequency and if there was relaxsation after each pressure.

Results: The effect of using feedback on the quality of pressure is the increase in the quality of chest compressions from 10 to over $50 \%$. The average depth of pressure without feedback was above the norm given in the guidelines, and after the hint exercise decreased to the normal value. The average frequency was within the normal range but as many as 40 out of 48 people who initially had an incorrect frequency with feedback were in the range of 100-120 per minute.

Conclusions: The use of an electrode with a pressure sensor significantly increased the quality of CPR conducted among both doctors and students, and the improvement was at a similar level in both groups. Interestingly, the results of students conducting CPR without hints were higher than doctors. It could be related to the fact that students were in the recently of first aid and/or emergency medicine classes. 